\title{
ON THE LIMITS OF QUOTIENTS OF POLYNOMIALS IN TWO VARIABLES
}

\author{
Roman Wituła, Edyta Hetmaniok ${ }^{1}$, Alicja Wróbel, Jarosław Matlak \\ Institute of Mathematics, Silesian University of Technology \\ Gliwice, Poland \\ 'edyta.hetmaniok@polsl.pl
}

\begin{abstract}
The aim of this paper is to discuss different types of decompositions and factorizations concerning a few families of symmetric polynomials in two variables including Ma polynomials, classic Cauchy polynomials, Ferrers-Jackson polynomials and some elementary polynomials as well. Application of the discussed decompositions and factorizations for determining the limits of quotients of the respective polynomials in two variables is presented here and some general theorems on these limits are also proven in this elaboration.
\end{abstract}

Keywords: Cauchy polynomials, Ma polynomials, Ferrers-Jackson polynomials limits of quotients of multivariable polynomials

\section{Introduction}

Xinrong Ma in [1] by using the Riordan's group theory has proven the following identity

$$
x^{n}+y^{n}+z^{n}=\sum_{k=0}^{\lfloor n / 3\rfloor} \frac{n}{n-2 k}\left(\begin{array}{c}
n-2 k \\
k
\end{array}\right)(x+y+z)^{n-3 k}(x y z)^{k},
$$

for every $x, y, z \in \mathbb{R}$, satisfying condition $x y+y z+z x=0$.

Since we have $z=-\frac{x y}{x+y}$ from the last condition, relation (1) can take the equivalent form

$$
\begin{aligned}
M_{n}(x, y) & =(x+y)^{n}\left(x^{n}+y^{n}\right)+(-x y)^{n} \\
& =\sum_{k=0}^{[n / 3]}(-1)^{k} \frac{n}{n-2 k}\left(\begin{array}{c}
n-2 k \\
k
\end{array}\right)\left(x^{2}+x y+y^{2}\right)^{n-3 k}(x y(x+y))^{2 k} .
\end{aligned}
$$

Polynomials $M_{n}(x, y), n \in \mathbb{N}$, will be called the Ma polynomials (see [2]).

Identity (2) is closely linked with two classic identities, the first one concerning decomposition of the Cauchy polynomials 


$$
\begin{aligned}
p_{n}(x, y): & =(x+y)^{2 n+1}-x^{2 n+1}-y^{2 n+1} \\
& =\sum_{k=0}^{\left[\frac{n-1}{3}\right]} \frac{2 n+1}{n-k}\left(\begin{array}{c}
n-k \\
2 k+1
\end{array}\right)\left(x^{2}+x y+y^{2}\right)^{n-1-3 k}(x y(x+y))^{2 k+1}
\end{aligned}
$$

and the second one concerning decomposition of the Ferrers-Jackson polynomials

$$
\begin{aligned}
q_{n}(x, y): & =(x+y)^{2 n}-x^{2 n}-y^{2 n} \\
& =\sum_{k=0}^{\left\lfloor\frac{n}{3}\right\rfloor} \frac{2 n}{n-k}\left(\begin{array}{c}
n-k \\
2 k
\end{array}\right)\left(x^{2}+x y+y^{2}\right)^{n-3 k}(x y(x+y))^{2 k} .
\end{aligned}
$$

Both identities in the present form have been proven by the authors in [3].

Ribenboim in [4] has presented the other decompositions of these polynomials (see chapter VII in [4]) together with their applications (for the solutions of some special cases of Fermat's Last Theorem).

The similarity of identity (2) to identities (3) and (4) is not a coincidence, the respective algebraic connections have been described in Theorem 1 of [2]. Among others, it has been proven there that

$$
\begin{gathered}
M_{2 n+1}(x, y)=\left(x^{2 n+1}+y^{2 n+1}\right) p_{n}(x, y)+x^{2(2 n+1)}+(x y)^{2 n+1}+y^{2(2 n+1)}, \\
M_{2 n}(x, y)=\left(x^{2 n}+y^{2 n}\right) q_{n}(x, y)+x^{4 n}+(x y)^{2 n}+y^{4 n} .
\end{gathered}
$$

In the current paper we also intend to discuss the decompositions and factorizations of polynomials belonging to two families of elementary symmetric polynomials - trinomials $E T_{n}^{ \pm}=x^{2 n} \pm(x y)^{n}+y^{2 n}$, and binomials $E B_{n}=x^{2 n}+y^{2 n}$, for every $n \in \mathbb{N}$.

The main goal of our considerations will be related to the applications of discussed here polynomials and their decompositions for generating the limits of quotients of the polynomials in two variables. It constitutes an essential strengthening of results obtained in paper [2]. In Section 1 we present an important theorem concerning the non-existence of the limits of quotients of polynomials in two variables together with a set of applications of this result to the distinguished above polynomials. In Section 2 we complete the decompositions given before with some new factorizations of polynomials and we show a number of their applications. Moreover, let us note that the presented paper essentially completes Ma's paper [1] and paper [3] of one of the authors, where similar type identities have been applied for the powers of elements of some, so called, conjugate recurrence sequences.

\section{Limits of quotients of polynomials in two variables - main negative result}

In this section we present some general results on the limits of quotients of polynomials in two variables. The main aim of introducing this theorem is the attempt to answer the question about the existence of limit 


$$
\lim _{(x, y) \rightarrow(0,0)} \frac{p(x, y)}{q(x, y)}
$$

in case when $p$ and $q$ represent different polynomials belonging to set

$$
\left\{p_{n}(x, y), q_{n}(x, y)-2, \quad M_{n}(x, y)-1, \quad E T_{2 n-1}^{ \pm}(x, y)\right\},
$$

for every $n \in \mathbb{N}$.

Now we present the announced theorem. First let us assume that $p, q \in \mathbb{R}[x, y]$, $(p, q)=1$ and the following decompositions hold

$$
p(x, y)=\sum_{i=0}^{k} p_{i}(x, y), \quad q(x, y)=\sum_{i=\lambda}^{l} q_{i}(x, y),
$$

where $p_{i}, q_{i}$ are the forms of degree $i$ and $q_{\lambda} \neq 0$.

Theorem 1. If either each of the following two inequalities $\pm q_{\lambda}(1, t)>0$ possesses a real solution or each of the following two inequalities $\pm q_{\lambda}(t, 1)>0$ possesses a real solution, then the finite limit (7) does not exist.

Proof. Without loss of generality of considerations one can take that for $t_{1}, t_{2} \in \mathbb{R}$ the inequalities $q_{\lambda}\left(1, t_{1}\right)>0>q_{\lambda}\left(1, t_{2}\right)$ are satisfied. By virtue of the taken assumption, for each subscript $i>\lambda$ the following relations hold

$$
q_{i}\left(x, t_{1} x\right)=O\left(|x|^{i}\right), \quad q_{i}\left(x, t_{2} x\right)=O\left(|x|^{i}\right) .
$$

Thus, there exists $\epsilon>0$ such that for each $x \in(0, \epsilon)$ we have

$$
q\left(x, t_{1} x\right)>0>q\left(x, t_{2} x\right) .
$$

Certainly, for each $x \in(0, \epsilon)$ the function $t \in \mathbb{R} \mapsto q(x, t x)$ is continuous and from (1) we get that between $t_{1}$ and $t_{2}$ there exists $t_{x}$ such that $q\left(x, t_{x} x\right)=0$.

Since $(p, q)=1$, the polynomials $p$ and $q$ possess only finitely many common zeros. In consequence, for sufficiently small $\epsilon>0$ we have $p\left(x, t_{x} x\right) \neq 0$, which means that

$$
\limsup _{(x, y) \rightarrow(0,0)} \frac{p(x, y)}{q(x, y)}=\infty
$$

Corollary 2. If $p \in \mathbb{R}[x, y]$ and $q$ belongs to set (8) for some $n \in \mathbb{N}$, then limit (7) does not exist.

Proof. It is sufficient to observe that

$$
\begin{aligned}
& p_{n}(1, t)=(2 n+1) t+\cdots, \quad q_{n}(1, t)-2=2 n t+\cdots, \\
& M_{n}(1, t)-1=n t+\cdots, \quad E T_{2 n-1}^{ \pm}(1, t)= \pm t^{2 n-1}+\cdots .
\end{aligned}
$$


Remark 3. Only a few papers exist (see for example $[5,6]$ ) devoted to the limits of quotients of some polynomials and even some functions of many variables, all of them are however very special, although they are certainly interesting. Our Theorem 1 is definitely better than theorems from cited papers, mostly for the reason of its general character, and also concerns the case of limits (7) for polynomials from set (8).

\section{Decomposition of some symmetric polynomials and its applications to the limits}

Obtained in [2] the divisibility relations of form

$$
\begin{array}{ccc}
\left(x^{2} \pm x y+y^{2}\right) \mid p_{n}(x, \pm y) & \Leftrightarrow & 3 \nmid(n-1), \\
\left(x^{2} \pm x y+y^{2}\right) \mid M_{n}(x, \pm y) & \Leftrightarrow & 3 \nmid n, \\
\left(x^{2} \pm x y+y^{2}\right) \mid q_{n}(x, \pm y) & \Leftrightarrow & 3 \nmid n, \\
\left(x^{2} \pm x y+y^{2}\right) \mid\left(x^{2 n}+(x y)^{n}+y^{2 n}\right) & \Leftrightarrow & 3 \nmid n, \\
\left(x^{2} \pm x y+y^{2}\right) \mid\left(x^{2 n}-(x y)^{n}+y^{2 n}\right) & \Leftrightarrow & 3 \nmid n \wedge 2 \nmid n
\end{array}
$$

lead to natural questions about factorization of such polynomials onto the factors of form $x^{2}+\omega x y+y^{2}$, where $\omega \in \mathbb{C}$.

First, we note that the following equality holds $(x, y \in \mathbb{C}$ and $n \in \mathbb{N})$ :

$$
x^{2 n}-(x y)^{n}+y^{2 n}=\prod_{\substack{1 \leq k \leq 3 n, \operatorname{gcd}(k, 6)=1}}\left(x^{2}-2 \cos \left(\frac{k \pi}{3 n}\right) x y+y^{2}\right) .
$$

This identity has been published by the authors in Mathematics Magazine (see [7]). For example, from (11) we obtain the identity

$$
\begin{gathered}
x^{8}-(x y)^{4}+y^{8}= \\
=\left(x^{2}-2 \cos \left(\frac{\pi}{12}\right) x y+y^{2}\right)\left(x^{2}-2 \cos \left(\frac{5 \pi}{12}\right) x y+y^{2}\right) \times \\
\quad \times\left(x^{2}-2 \cos \left(\frac{7 \pi}{12}\right) x y+y^{2}\right)\left(x^{2}-2 \cos \left(\frac{11 \pi}{12}\right) x y+y^{2}\right),
\end{gathered}
$$

where

$$
\begin{aligned}
& 2 \cos \left(\frac{\pi}{12}\right)=-2 \cos \left(\frac{11 \pi}{12}\right)=\sqrt{2+\sqrt{3}}=\frac{\sqrt{6}+\sqrt{2}}{2}, \\
& 2 \sin \left(\frac{\pi}{12}\right)=2 \cos \left(\frac{5 \pi}{12}\right)=-2 \cos \left(\frac{7 \pi}{12}\right)=\sqrt{2-\sqrt{3}}=\frac{\sqrt{6}-\sqrt{2}}{2} .
\end{aligned}
$$

Hence, for example, for arbitrary $r>0$ and $\alpha \in \mathbb{C}$ we get the limits 


$$
\begin{gathered}
\lim _{\substack{x^{2}-2 \cos \left(\frac{\pi}{12}\right) x y+y^{2} \rightarrow 0 \\
x, y \in \mathbb{C},|x y| \geq r}} \frac{x^{8}-(x y)^{4}+y^{8}}{(x y)^{3}\left(x^{2}-2 \cos \left(\frac{\pi}{12}\right) x y+y^{2}\right)}= \\
=\lim _{\substack{A:=x^{2}-2 \cos \left(\frac{\pi}{12}\right) x y+y^{2} \rightarrow 0 \\
x, y \in \mathbb{C},|x y| \geq r}} \frac{1}{(x y)^{3}}\left(A+2\left(\cos \left(\frac{\pi}{12}\right)-\cos \left(\frac{5 \pi}{12}\right)\right) x y\right) \times \\
\times\left(A+2\left(\cos \left(\frac{\pi}{12}\right)-\cos \left(\frac{7 \pi}{12}\right)\right) x y\right)\left(A+2\left(\cos \left(\frac{\pi}{12}\right)-\cos \left(\frac{11 \pi}{12}\right)\right) x y\right)= \\
=2 \sqrt{2+\sqrt{3}}(\sqrt{2+\sqrt{3}}-\sqrt{2-\sqrt{3}})(\sqrt{2+\sqrt{3}}+\sqrt{2-\sqrt{3}})=4 \sqrt{6+3 \sqrt{3}}
\end{gathered}
$$

and

$$
\begin{gathered}
\lim _{\substack{x, y \in \mathbb{C} \backslash\{0\} \\
\frac{x}{y}+\frac{y}{x} \rightarrow \alpha}} \frac{x^{8}-(x y)^{4}+y^{8}}{(x y)^{4}}= \\
=\lim _{\substack{x, y \in \mathbb{C}\left\{\{0\} \\
B:=\frac{x^{2}-\alpha x y+y^{2}}{x y} \rightarrow 0\right.}} \frac{1}{(x y)^{3}}\left(B+\alpha-2 \cos \left(\frac{\pi}{12}\right)\right)\left(B+\alpha-2 \cos \left(\frac{5 \pi}{12}\right)\right) \times \\
\times\left(B+\alpha-2 \cos \left(\frac{7 \pi}{12}\right)\right)\left(B+\alpha-2 \cos \left(\frac{11 \pi}{12}\right)\right)= \\
=(\alpha-\sqrt{2+\sqrt{3}})(\alpha+\sqrt{2+\sqrt{3}})(\alpha-\sqrt{2-\sqrt{3}})(\alpha+\sqrt{2-\sqrt{3}})= \\
=\alpha^{4}-2 \alpha^{2}+1 .
\end{gathered}
$$

Remark 4. We also have the following decomposition:

$$
\begin{gathered}
x^{4}+3 x^{2} y^{2}+y^{4}=\left(x^{2}+\alpha^{2} y^{2}\right)\left(x^{2}+\beta^{2} y^{2}\right) \\
=(x+i \alpha y)(x-i \alpha y)(x+i \beta y)(x-i \beta y),
\end{gathered}
$$

where $\alpha=\frac{1+\sqrt{5}}{2}$ and $\beta=\frac{1-\sqrt{5}}{2}$ (we remember that $\alpha$ is the golden ratio).

Hence, for example, we obtain that

$$
\begin{aligned}
\lim _{\substack{x, y \in \mathbb{C} \backslash\{0\} \\
\frac{x}{y} \rightarrow i \alpha}} \frac{x^{4}+3 x^{2} y^{2}+y^{4}}{y^{3}(x-i \alpha y)} & =\lim _{\substack{x, y \in \mathbb{C} \backslash\{0\} \\
\frac{x}{y} \rightarrow i \alpha}}\left(\frac{x}{y}+i \alpha\right)\left(\frac{x^{2}}{y^{2}}+\beta^{2}\right) \\
& =2 i \alpha\left(\beta^{2}-\alpha^{2}\right)=-i(5+\sqrt{5}) .
\end{aligned}
$$

Moreover, we discover two other interesting decompositions (see [8]):

$$
\begin{aligned}
& x^{4}+4 x^{2} y^{2}+y^{4}=\left(x^{2}+4 y^{2} \sin ^{2} \frac{\pi}{12}\right)\left(x^{2}+4 y^{2} \cos ^{2} \frac{\pi}{12}\right) \\
& =\left(x+2 i y \sin \frac{\pi}{12}\right)\left(x-2 i y \sin \frac{\pi}{12}\right)\left(x+2 i y \cos \frac{\pi}{12}\right)\left(x-2 i y \cos \frac{\pi}{12}\right),
\end{aligned}
$$


where $\cos \frac{\pi}{12}=\frac{\sqrt{6}+\sqrt{2}}{4}, \sin \frac{\pi}{12}=\frac{\sqrt{6}-\sqrt{2}}{4}$ and

$$
\begin{gathered}
x^{4}+11 x^{2} y^{2}+y^{4}=\left(x^{2}+64 y^{2} \cos ^{2} \frac{4 \pi}{13} \cos ^{2} \frac{10 \pi}{13} \cos ^{2} \frac{12 \pi}{13}\right) \times \\
\times\left(x^{2}+64 y^{2} \cos ^{2} \frac{2 \pi}{13} \cos ^{2} \frac{6 \pi}{13} \cos ^{2} \frac{8 \pi}{13}\right) \\
=\left(x+i y \frac{\sqrt{13}+3}{2}\right)\left(x-i y \frac{\sqrt{13}+3}{2}\right)\left(x+i y \frac{\sqrt{13}-3}{2}\right)\left(x-i y \frac{\sqrt{13}-3}{2}\right),
\end{gathered}
$$

which are the special cases of the following formula (the product below is taken over all four combinations of signs + and - ):

$$
\begin{aligned}
& \prod\left(x \pm i \frac{y}{2}\left(\sqrt{a^{2}+b^{2}} \pm a\right)\right)=\left(x^{2}+\frac{y^{2}}{4}\left(\sqrt{a^{2}+b^{2}}+a\right)^{2}\right) \times \\
& \quad \times\left(x^{2}+\frac{y^{2}}{4}\left(\sqrt{a^{2}+b^{2}}-a\right)^{2}\right)=x^{4}+(x y)^{2}\left(a^{2}+\frac{1}{2} b^{2}\right)+y^{4} \frac{b^{4}}{16}
\end{aligned}
$$

Hence we get

$$
\lim _{\frac{x}{y} \rightarrow i \frac{\sqrt{a^{2}+b^{2}}-a}{2}} \frac{x^{4}+(x y)^{2}\left(a^{2}+\frac{1}{2} b^{2}\right)+y^{4} \frac{b^{4}}{16}}{y^{3}\left(x-i y \frac{\sqrt{a^{2}+b^{2}}-a}{2}\right)}=i a\left(a^{2}+b^{2}-a \sqrt{a^{2}+b^{2}}\right),
$$

which is the generalization of (13).

Remark 5. We note that decomposition (11) is equivalent to the following two decompositions

$$
x^{2 n}+(x y)^{n}+y^{2 n}=\prod_{\substack{1 \leq k \leq 3 n, \operatorname{gcd}(k, 6)=1}}\left(x^{2}+2 \cos \left(\frac{k \pi}{3 n}\right) x y+y^{2}\right),
$$

whenever $n$ is odd, and

$$
x^{2 n}+(x y)^{n}+y^{2 n}=\prod_{\substack{1 \leq k \leq 3 n, \operatorname{gcd}(k, 6)=1}}\left(\left(e_{v} x\right)^{2}+2 e_{v} \cos \left(\frac{k \pi}{3 n}\right) x y+y^{2}\right)
$$

where $e_{v}:=\exp \left(\frac{i \pi}{2 v}\right), v:=\operatorname{val}_{2}(n)$, i.e. $v:=\max \left\{k \in \mathbb{N}: 2^{k} \mid n\right\}$, whenever $n$ is even. For example, we obtain 


$$
\begin{aligned}
x^{8}+ & (x y)^{4}+y^{8}= \\
= & \left(i x^{2}-2 e^{i \pi / 4} \cos \left(\frac{\pi}{12}\right) x y+y^{2}\right)\left(i x^{2}-2 e^{i \pi / 4} \cos \left(\frac{5 \pi}{12}\right) x y+y^{2}\right) \\
& \times\left(i x^{2}-2 e^{i \pi / 4} \cos \left(\frac{7 \pi}{12}\right) x y+y^{2}\right)\left(i x^{2}-2 e^{i \pi / 4} \cos \left(\frac{11 \pi}{12}\right) x y+y^{2}\right)= \\
=- & \left(e^{i \pi / 4} x^{2}-2 \cos \left(\frac{\pi}{12}\right) x y+e^{-i \pi / 4} y^{2}\right)\left(e^{i \pi / 4} x^{2}-2 \cos \left(\frac{5 \pi}{12}\right) x y+e^{-i \pi / 4} y^{2}\right) \\
\times & \left(e^{i \pi / 4} x^{2}-2 \cos \left(\frac{7 \pi}{12}\right) x y+e^{-i \pi / 4} y^{2}\right)\left(e^{i \pi / 4} x^{2}-2 \cos \left(\frac{11 \pi}{12}\right) x y+e^{-i \pi / 4} y^{2}\right) .
\end{aligned}
$$

But, we also get the following decompositions

$$
\begin{gathered}
x^{4}+(x y)^{2}+y^{4} \quad=\left(x^{2}+x y+y^{2}\right)\left(x^{2}-x y+y^{2}\right) \\
=\left(x^{2}-i \sqrt{3} x y-y^{2}\right)\left(x^{2}+i \sqrt{3} x y-y^{2}\right), \\
x^{8}+(x y)^{4}+y^{8}=\left(x^{4}+y^{4}\right)^{2}-(x y)^{4} \\
=\left(x^{4}-(x y)^{2}+y^{4}\right)\left(x^{4}+(x y)^{2}+y^{4}\right) \\
=\left(x^{2}-\sqrt{3} x y+y^{2}\right)\left(x^{2}+\sqrt{3} x y+y^{2}\right)\left(x^{2}+x y+y^{2}\right)\left(x^{2}-x y+y^{2}\right),
\end{gathered}
$$

which means that in case of even $n$ there also exists the other decomposition of polynomial $x^{2 n}+(x y)^{n}+y^{2 n}$.

We prove that

$$
x^{2 n}+(x y)^{n}+y^{2 n}=\prod_{k=1}^{n}\left(x^{2}-2 \cos \left(\frac{2(3 k+1) \pi}{3 n}\right) x y+y^{2}\right)
$$

for every $n \in \mathbb{N}$. To this aim we note that

$$
x^{2 n}+(x y)^{n}+y^{2 n}=0 \Leftrightarrow x^{n}=e^{\left( \pm \frac{2}{3} \pi i\right)} y^{n} \Leftrightarrow x=y \exp \left(\frac{2(3 k \pm 1)}{3 n} \pi i\right),
$$

for every $k=1,2, \ldots, n$. Furthermore, we have

$$
\begin{gathered}
\left(x-y \exp \left(\frac{2(3 k+1)}{3 n} \pi i\right)\right)\left(x-y \exp \left(\frac{2(3(n-k)-1)}{3 n} \pi i\right)\right) \\
=x^{2}-x y\left(\exp \left(\frac{2(3 k+1)}{3 n} \pi i\right)+\exp \left(\frac{2(-3 k-1)}{3 n} \pi i\right)\right)+y^{2} \\
=x^{2}-2 x y\left(\cos \left(\frac{2(3 k+1)}{3 n} \pi\right)\right)+y^{2},
\end{gathered}
$$

which implies (18). Moreover, from (18) and from the following decomposition

$$
\begin{aligned}
x^{4(2 n+1)}+(x y)^{2(2 n+1)}+y^{4(2 n+1)}= & \left(x^{2(2 n+1)}-(x y)^{2 n+1}+y^{2(2 n+1)}\right) \times \\
& \times\left(x^{2(2 n+1)}+(x y)^{2 n+1}+y^{2(2 n+1)}\right),
\end{aligned}
$$


we deduce that

$$
=\prod_{\substack{1 \leq k \leq 3(2 n+1) \\ \operatorname{gcd}(k, 6)=1}}\left(x^{2}-2 x y \cos \frac{k \pi}{3(2 n+1)}+y^{2}\right)\left(x^{2}+2 x y \cos \frac{k \pi}{3(2 n+1)}+y^{2}\right) .
$$

For example, we obtain

$$
\begin{aligned}
& \left\{\lim _{\substack{x, y \in \mathbb{C} \backslash\{0\} \\
\frac{x}{y} \rightarrow \exp \left(i \pi\left(\frac{1}{2}-\frac{1}{3(2 n+1)}\right)\right)}} \frac{x^{2(2 n+1)}+(x y)^{(2 n+1)}+y^{2(2 n+1)}}{y^{4 n}\left(x^{2}-2\left(\sin \frac{\pi}{3(2 n+1)}\right) x y+y^{2}\right)}=\right. \\
= & \left\{\lim _{x, y \in \mathbb{C} \backslash\{0\}} \prod_{k=1}^{n-1}\left(\left(\frac{x}{y}\right)^{2}-2\left(\cos \frac{2(3 k+1) \pi}{3(2 n+1)}\right) \frac{x}{y}+1\right)\right. \\
= & (2 i)^{n-1} e^{i \pi\left(\frac{x}{2(2 n+1)}-\frac{1}{6}\right)} \prod_{k=1}^{n-1}\left(\sin \frac{\pi}{3(2 n+1)}-\cos \frac{2(3 k+1) \pi}{3(2 n+1)}\right) .
\end{aligned}
$$

Remark 6. We note also that in [3] the following decomposition has been proven

$$
x^{2 n}+y^{2 n}=\sum_{r=0}^{n} w_{r, n}(x y)^{r}\left(x^{2}+x y+y^{2}\right)^{n-r},
$$

where

$$
w_{r, n}=\sum_{l=0}^{r}(-1)^{l} \frac{2 n}{2 n-l}\left(\begin{array}{c}
2 n-l \\
l
\end{array}\right)\left(\begin{array}{l}
n-l \\
r-l
\end{array}\right), \quad r=0,1, \ldots, n .
$$

One can generate the following recurrence relations

$$
\left\{\begin{array}{c}
w_{0, n}=w_{0, n+1}, \\
w_{1, n}-w_{0, n}=w_{1, n+1}, \\
w_{r, n}-w_{r-1, n}-w_{r-2, n-1}=w_{r, n+1}, \quad r=2, \ldots, n \\
-w_{n, n}-w_{n-1, n-1}=w_{n+1, n+1}
\end{array}\right.
$$

It is easy to deduce the identity of the form $w_{n, n}=2 \cos \left(\frac{2}{3} \pi n\right)$, i.e. the identity given below holds

$$
\sum_{l=0}^{n} \frac{(-1)^{l}}{2 n-l}\left(\begin{array}{c}
2 n-l \\
l
\end{array}\right)=\frac{1}{n} \cos \left(\frac{2}{3} \pi n\right) .
$$

On the other hand, elements $w_{k, n}$, for $k \ll n$, are the polynomials of variable $n$ with rational coefficients of degree $k$, i.e. the following relation holds true 


$$
\begin{aligned}
(-1)^{k} k ! w_{k, n}=p_{k}(n), \quad p_{k} \in \mathbb{Q}[n], \quad \operatorname{deg} p_{k}=k, & \underline{\frac{4}{4}(27 k-25)\left(\begin{array}{l}
k \\
3
\end{array}\right)} n^{k-2} \\
p_{k}(n)= & n^{k}-3\left(\begin{array}{l}
k \\
2
\end{array}\right) n^{k-1}+\left(\begin{array}{l}
k \\
4
\end{array}\right) n^{k-3} \ldots .
\end{aligned}
$$

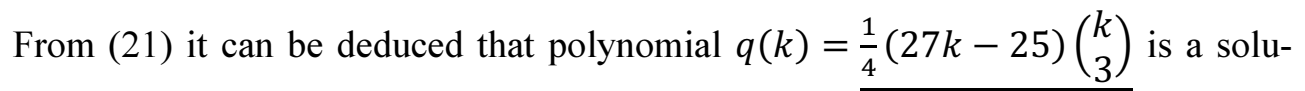
tion of the recurrence relation

$$
\begin{gathered}
q(k)(k-2)-q(k-1) k= \\
=5\left(\begin{array}{l}
k \\
3
\end{array}\right)+\frac{9}{2}(k-1)\left(\begin{array}{l}
k \\
3
\end{array}\right)+9(k-3)\left(\begin{array}{l}
k \\
3
\end{array}\right)=\frac{1}{2}(27 k-25)\left(\begin{array}{l}
k \\
3
\end{array}\right) .
\end{gathered}
$$

Moreover, from (20) for $n \geq 6$ we obtain (the calculations were made in Mathematica v.9 software):

$$
\begin{aligned}
& p_{1}(n)=1, \quad p_{2}(n)=(n-3) n, \quad p_{3}(n)=(n-7)(n-2) n, \\
& p_{4}(n)=(n-3) n\left(n^{2}-15 n+38\right), \quad p_{5}(n)=(n-17)(n-6)(n-4)(n-3) n, \\
& p_{6}(n)=(n-5)(n-4) n\left(-846+341 n-36 n^{2}+n^{3}\right), \\
& p_{7}(n)=(n-6)(n-5)(n-4) n\left(-2358+641 n-48 n^{2}+n^{3}\right), \\
& p_{8}(n)=n(n-5)(n-6)(n-7)(n-9)(n-18)\left(n^{2}-39 n+164\right) .
\end{aligned}
$$

Let us also present a few of the next formulae, in some other form, as the limits of quotients of the respective polynomials

$$
\begin{array}{ll}
\lim _{x \rightarrow 0} \frac{p_{15}(x)}{p_{14}(x)}=\frac{842166}{1437863}, & \lim _{x \rightarrow 0} \frac{p_{13}(x)}{p_{12}(x)}=\frac{2555292}{73789}, \\
\lim _{x \rightarrow 0} \frac{p_{12}(x)}{p_{11}(x)}=-11 \frac{73789}{25653}, & \lim _{x \rightarrow 0} \frac{p_{13}(x)}{p_{12}(x)}=\frac{2555292}{73789},
\end{array}
$$

It is worth emphasizing that the Mathematica software has proposed one more relation, using the hypergeometric function, of the form

$$
w_{n-k, n}=\left(\begin{array}{c}
n \\
n-k
\end{array}\right) \cdot{ }_{2} \mathrm{~F}_{1}\left(\frac{1}{2}-n, k-n ; 1-2 n ; 4\right),
$$

which is however "compatible" with the basing definition of function $w_{n-k, n}$ (in the first impulse as the surprised researchers we were full of hope for applying some of the identities included, for example, in the newest paper like [9]).

Furthermore, like it has been generated in [3], we have

$$
w_{n-1, n}=\frac{2}{3} \sqrt{3} n \sin \left(\frac{2}{3} \pi n\right), \quad w_{n-2, n}=-\frac{n^{2}}{3} \cos \left(\frac{2}{3} \pi n\right)-\frac{\sqrt{3}}{9} \sin \left(\frac{2}{3} \pi n\right) .
$$


Hence and from (20) we obtain

$$
\begin{gathered}
w_{n-1, n}=\sum_{l=0}^{n-1}(-1)^{l} \frac{2 n}{2 n-l}\left(\begin{array}{c}
2 n-l \\
l
\end{array}\right)(n-l) \\
=n\left(2(-1)^{n-1}+\sum_{l=0}^{n}(-1)^{l} \frac{2 n}{2 n-l}\left(\begin{array}{c}
2 n-l \\
l
\end{array}\right)-\sum_{l=0}^{n-1}(-1)^{l} \frac{2 l}{2 n-l}\left(\begin{array}{c}
2 n-l \\
l
\end{array}\right)\right) \\
=n\left(2(-1)^{n-1}+\cos \left(\frac{2}{3} \pi n\right)-\sum_{l=1}^{n-1}(-1)^{l}\left(\begin{array}{c}
2 n-l-1 \\
l-1
\end{array}\right)\right)=\frac{2}{3} \sqrt{3} n \sin \left(\frac{2}{3} \pi n\right),
\end{gathered}
$$

which implies

$$
\begin{aligned}
\sum_{l=1}^{n}(-1)^{l}\left(\begin{array}{c}
2 n-l-1 \\
l-1
\end{array}\right)=(-1)^{n-1} & +\cos \left(\frac{2}{3} \pi n\right)-\frac{2}{3} \sqrt{3} n \sin \left(\frac{2}{3} \pi n\right)= \\
= & (-1)^{n-1}+\sqrt{\frac{7}{3}} \cos \left(\frac{2}{3} \pi n+\varphi\right)
\end{aligned}
$$

where $\varphi:=\arcsin \left(\frac{2}{7} \sqrt{7}\right)$.

Let us notice that from identity (19) the following fact can be also deduced.

Theorem 7. If $n \geq 2, p \in \mathbb{R}$ and $r \in(0,1)$ then

$$
\begin{gathered}
\lim _{\substack{x, y \rightarrow 0, x, y \in \mathbb{C},|x|+|y|>0, x \neq 0 \Rightarrow\left|\frac{y}{x}-e^{ \pm i 2 \pi / 3}\right|>r, y \neq 0 \Rightarrow\left|\frac{x}{y}-e^{ \pm i 2 \pi / 3}\right|>r}} \frac{x^{2 n}+y^{2 n}-2 \cos \left(\frac{2}{3} \pi n\right)(x y)^{n}}{\left(x^{2}+x y+y^{2}\right)^{p}}= \\
=\left\{\begin{array}{cl}
0, & \text { whenever } p<n, \\
\text { does not exist } & \text { whenever } p \geq n .
\end{array}\right.
\end{gathered}
$$

Proof. From (19) we obtain (for $n \geq 2$ ):

$$
\begin{gathered}
\frac{x^{2 n}+y^{2 n}-2 \cos \left(\frac{2}{3} \pi n\right)(x y)^{n}}{\left(x^{2}+x y+y^{2}\right)^{p}}=\left(\begin{array}{cc}
\text { for } & |x| \geq|y| \\
\text { and } & |x|+|y|>0
\end{array}\right) \\
=x^{2(n-p)} \sum_{r=0}^{n-1} w_{r, n} \frac{\left(\frac{y}{x}\right)^{r}}{\left(1+\frac{y}{x}+\left(\frac{y}{x}\right)^{2}\right)^{p-n+r}} .
\end{gathered}
$$

Hence, if $p<n$ and

$$
x \neq 0 \Rightarrow\left|\frac{y}{x}-e^{ \pm i 2 \pi / 3}\right|>r \quad \text { and } \quad y \neq 0 \Rightarrow\left|\frac{x}{y}-e^{ \pm i 2 \pi / 3}\right|>r,
$$

then the respective limit is equal to zero. 
If $p>n$ then liminf and limsup of the discussed fraction are equal to 0 and $\infty$, respectively. Finally, if $p=n$ and $\lim \frac{y}{x}=\beta \in \mathbb{R}$, then the limit is equal to

$$
\sum_{r=0}^{n-1} w_{r, n} \frac{\beta^{r}}{\left(1+\beta+\beta^{2}\right)^{r}}
$$

thus, the respective limit does not exist (since $\beta \in \mathbb{R}$ ).

Moreover, from (19) the following special limits can be calculated (all of them are discussed in the complex domain):

$$
\begin{gathered}
\lim _{\substack{x \rightarrow \alpha \in \mathbb{C} \\
x^{2}+x y+y^{2} \rightarrow 0}} \frac{x^{10}+(x y)^{5}+y^{10}}{y\left(x^{2}+x y+y^{2}\right)}=-5 \alpha^{7}, \\
\lim _{\substack{x \rightarrow \alpha \in \mathbb{C} \\
x^{2}+x y+y^{2} \rightarrow 0}} \frac{x^{14}+(x y)^{7}+y^{14}}{x^{2}+x y+y^{2}}=7 \alpha^{12}, \\
\lim _{\substack{x \rightarrow \alpha \in \mathbb{C} \\
y \rightarrow-\alpha}} \frac{x^{10}+(x y)^{5}+y^{10}-\left(x^{2}+x y+y^{2}\right)^{5}}{(x+y)^{2}}=20 \alpha^{8}, \\
\lim _{\substack{x \rightarrow \alpha \in \mathbb{C} \\
y \rightarrow-\alpha}} \frac{x^{14}+(x y)^{7}+y^{14}-\left(x^{2}+x y+y^{2}\right)^{7}}{(x+y)^{2}}=42 \alpha^{12}, \\
\lim _{\substack{x, y \in \mathbb{C} \\
x^{2}+x y+y^{2} \rightarrow 0 \\
(x y)^{2} \rightarrow \alpha \neq 0}} \frac{x^{14}+(x y)^{7}+y^{14}-\left(x^{2}+x y+y^{2}\right)^{7}}{x^{10}+(x y)^{5}+y^{10}-\left(x^{2}+x y+y^{2}\right)^{5}}=-\frac{7}{5} \alpha \\
x^{2}
\end{gathered}
$$

and the last one, for the contrast considered in real domain,

$$
\lim _{\substack{x, y \in \mathbb{R} \\ x, y \rightarrow 0}} \frac{x^{14}+(x y)^{7}+y^{14}-\left(x^{2}+x y+y^{2}\right)^{7}}{x^{10}+(x y)^{5}+y^{10}-\left(x^{2}+x y+y^{2}\right)^{5}}=0
$$

(in the last case the limit is equal to zero also under weakened assumptions, that is, if $x, y \in \mathbb{C}, x, y \rightarrow 0$ and $(x y) /\left(x^{2}+y^{2}\right)$ is bounded), since we have (see [3]):

$$
\begin{gathered}
x^{10}+(x y)^{5}+y^{10}=\left(x^{2}+x y+y^{2}\right)^{5}-5 x y\left(x^{2}+x y+y^{2}\right)^{4} \\
+5(x y)^{2}\left(x^{2}+x y+y^{2}\right)^{3}+5(x y)^{3}\left(x^{2}+x y+y^{2}\right)^{2}-5(x y)^{4}\left(x^{2}+x y+y^{2}\right) \\
=\left(x^{2}+x y+y^{2}\right)^{5}-5 x y\left(x^{2}+x y+y^{2}\right)\left(x^{2}+y^{2}\right)^{2} \\
x^{14}+(x y)^{7}+y^{14}= \\
=\left(x^{2}+x y+y^{2}\right)^{7}-7 x y\left(x^{2}+x y+y^{2}\right)^{6}+14(x y)^{2}\left(x^{2}+x y+y^{2}\right)^{5} \\
-21(x y)^{4}\left(x^{2}+x y+y^{2}\right)^{3}+7(x y)^{5}\left(x^{2}+x y+y^{2}\right)^{2}+7(x y)^{6}\left(x^{2}+x y+y^{2}\right) \\
=\left(x^{2}+x y+y^{2}\right)^{7}-7 x y(x+y)^{2}\left(x^{2}+x y+y^{2}\right)\left(x^{2}+y^{2}\right) \\
\times\left(\left(\left(x^{2}+x y+y^{2}\right)^{2}+(x y)^{2}\right)(x+y)^{2}-3 x y\left(x^{2}+x y+y^{2}\right)^{2}\right) .
\end{gathered}
$$


From the last equalities in both above formulae we obtain

$$
\begin{gathered}
\frac{x^{14}+(x y)^{7}+y^{14}-\left(x^{2}+x y+y^{2}\right)^{7}}{x^{10}+(x y)^{5}+y^{10}-\left(x^{2}+x y+y^{2}\right)^{5}}= \\
=\frac{7}{5} \cdot \frac{x^{6}+y^{6}+x y\left(x^{4}+y^{4}\right)+3 x^{2} y^{2}\left(x^{2}+x y+y^{2}\right)}{x^{2}+y^{2}} .
\end{gathered}
$$

For contrast we note that the following nice identity holds

$$
\frac{x^{10}+(x y)^{5}+y^{10}-\left(x^{2}+x y+y^{2}\right)^{5}}{x^{6}+(x y)^{3}+y^{6}-\left(x^{2}+x y+y^{2}\right)^{3}}=\frac{5}{3}\left(x^{2}+y^{2}\right)\left(x^{2}+x y+y^{2}\right) .
$$

\section{References}

[1] Ma X., A generalization of the Kummer identity and its application to Fibonacci-Lucas sequences, The Fibonacci Quarterly 1998, 36, 339-347.

[2] Wituła R., Hetmaniok E., Słota D., Problem 1876, Math. Magazine October 2011 issue.

[3] Wituła R., Słota D., Cauchy, Ferrers-Jackson and Chebyshev polynomials and identities for the powers of elements of some conjugate recurrence sequences, Central Eur. J. Math. 2006, 4, 531-546 .

[4] Ribenboim P., Fermat's Last Theorem for Amateurs, Springer-Verlag, New York Inc., 1999.

[5] Liu Y., Wang Y., A class of multivariable limits, College Math. Journal 2010, 41, 2, 194-196.

[6] Choi J., Rathie A.K, New results for the series ${ }_{2} F_{1}(x)$ with an application, Commun. Korean Math. Soc., 2014, 29, 65-74.

[7] Wituła R., On some applications of formulae for the sum of unimodular complex numbers, Pracownia Komputerowa Jacka Skalmierskiego, Gliwice 2011.

[8] Vajda S., Fibonacci and Lucas Numbers, and the Golden Section, Theory and Application, Dover Publications, Inc., 2008.

[9] Hayashi N., Matusi Y., Decomposition formulae for generalized hypergeometric functions with the Gauss - Kummer identity, Commun. Korean Math. Soc., 2014, 29, 97-108.

[10] Prudnikov A.P., Brychkov Y.A., Marichev O.I, Integrals and series. 1. Elementary functions, translated from the Russian and with a preface by N.M. Queen, Gordon \& Breach Science Publishers, New York 1986.

[11] Wituła R., Hetmaniok E., Słota D., Ma’s identity and its applications, Annales Universitatis Paedagogicae Cracoviensis 2012, 11, 43-51.

[12] Schuette P., A question of limits, Math. Magazine 2004, 77, 1, 61-68. 